\title{
Aplicación de algunas ideas del utopismo clásico a la creación de una matriz política global que permita el desacuerdo, la pluralidad y la diversidad organizativa
}

\author{
Samuel Gallastegui \\ Florida Universitaria, s.gallastegui@florida-uni.es
}

\begin{abstract}
This research proposal focuses on the problem produced by the hegemony of capitalism mechanics, which constrains the power and effectiveness of the many political matrices that are the nations of the world, resulting incapable to solve global social and ecological problems. I hypothesize the development of a global political matrix that share common political and ethical principles, but that at the same time allows different models and mechanisms of social and economic organization, and to achive it $i$ have applied and developed some ideas of classical utopianism. As a result I have found the initial difficulty that classical models are shown as perfect and homogeneous, which is against human diversity itself and ethical pluralism. However, I have developed some ideas drawn from these utopian models that would create a system that admits diversity, plurality and disagreement.
\end{abstract}

Keywords: Politics, Hegemony, Global, Utopia, Diversity

\begin{abstract}
Resumen
Esta propuesta de investigación se centra en el problema producido por la hegemonía del mecanismo capitalista, que limita el poder y la eficacia de las matrices políticas que forman las naciones del mundo, resultando estas incapaces de resolver los problemas sociales y ecológicos globales. Planteo la hipótesis del desarrollo de una matriz política global que comparta principios políticos y éticos comunes, pero que al mismo tiempo permita diferentes modelos y mecanismos de organización social y económica; y para lograrlo he aplicado y desarrollado algunas ideas del utopismo clásico. Como resultado, he encontrado la dificultad inicial de que los modelos clásicos se muestran perfectos y homogéneos, lo que va en contra de la diversidad humana y del pluralismo ético. Sin embargo, he desarrollado algunas ideas extraídas de estos modelos utópicos que sí pudieran dar lugar a un sistema que admita la diversidad organizativa, la pluralidad y el desacuerdo.
\end{abstract}

Palabras clave: Política, Hegemonía, Global, Utopía, Diversidad 


\section{Introducción y objetivos}

La comunidad humana se enfrenta a retos sin precedentes en su historia. La contaminación producida por las actividades de producción puede estar generando cambios peligrosos en la configuración del planeta que requieren una respuesta conjunta. Además, las tecnologías de la información eliminan progresivamente los límites mentales en todo el planeta, creando así una sensación de convivencia de toda la humanidad. No han borrado, sin embargo, los límites físicos y jurídicos, creando de facto una sociedad de estrados en la que ciudadanos que conviven tienen derechos diferentes, tanto en la norma como en la práctica.

Desde la caída del muro de Berlín, el mecanismo capitalista se ha impuesto sobre cualquier otra forma de ordenación de la actividad productiva y política. (Herrera Guillén, 2013, 91-93). El mercado como mecanismo regulador ha quedado asumido como un sistema hegemónico por encima de las matrices políticas (Gill y Law, 1989) que mejora la calidad de vida de las personas e impulsa el progreso y el dominio de la naturaleza, más allá de lo que imaginara Bacon (1980). Sin embargo, en el camino ha tenido que renunciar a los máximos de autonomía que le son propias al progreso material en tanto que utopía.

El poder político ha quedado constreñido en una escala local y con un margen muy limitado para establecer alternativas, pero los problemas políticos a los que nos enfrentamos conjuntamente tienen una escala planetaria. Se hace necesario un modelo de poder y organización política global que sea efectiva para enfrentar estos retos al tiempo que permita otras formas de organización dentro de sí, conciliando así unidad y discrepancia, igualdad y heterogeneidad, de forma tal que la actual conformidad en diferencia (un mismo sistema compartido por personas en desigualdad de condiciones) pudiera convertirse en una igualdad en la disconformidad (personas en igualdad de condiciones pero diferentes formas de organizarse económica y políticamente).

En el pasado han emergido propuestas que dialogaban con los modelos de organización política de su tiempo mediante la propuesta de un modelo alternativo, entre los que se destacan por su carácter fundacional las utopías del Renacimiento. De todas maneras, si el modelo alternativo a la actual situación global fuera una utopía, esta debería ser poliutópica, es decir, unos principios comunes que permitieran diferentes modelos de organización social y política; o una matriz política en la que se pudieran insertar diferentes modelos, conciliando igualdad (derechos, libertades, dignidad) y heterogeneidad (pensamientos, corrientes, modelos políticos y económicos).

\section{Hipótesis y método}

El sistema económico actúa como un único mecanismo regulador en el que se insertan diversas matrices políticas. Este artículo plantea una aproximación teórica que invierta la figura, esto es, una matriz política única a nivel global en la que se puedan insertar diferentes mecanismos socioeconómicos, de forma que permita el desacuerdo, la pluralidad y la diversidad organizativa, al tiempo que capacite al poder político para enfrentar los grandes retos planetarios.
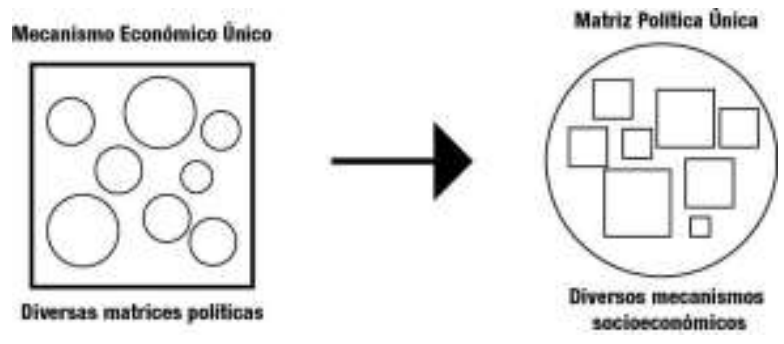

Fig. 1. Gráfico. Inversión de las estructuras políticas y económicas

Platón, Moro o Campanella, entre otros, presentaron en su momento propuestas alternativas a los modelos de su época, revelando así sus fallos y cómo enmendarlos. Aunque los modelos clásicos tienen una forma aparentemente estática, perfecta y homogénea, podemos extraer las condiciones formales que permitan crear un nuevo modelo "poliutópico": un modelo abierto, imperfecto y en continuo cambio surgido de algunas ideas esenciales del utopismo clásico. Estas pueden funcionar como los principios permanentes y estables que fundamenten la diversidad. 


\section{Desarrollo}

Parecería que la falta de desacuerdo y pluralidad debilita a la larga un sistema político. Sin embargo, los utopistas clásicos ofrecen modelos demasiado uniformes y acabados que no permiten la integración de otras formas organizativas, en tanto que presentan una única solución teóricamente perfecta. Aunque estos dos aspectos (modelo perfecto / obra en progreso y homogeneidad/ heterogeneidad) difieren del utopismo clásico, sus textos pueden ser de gran ayuda para sentar las bases en las que construir un modelo global que concilie igualdad y heterogeneidad, una matriz política global en la que se puedan ensamblar diferentes formas de organización. He revisado las utopías del Renacimiento, especialmente las de Moro y Campanella, para buscar ciertas ideas prácticas en las que basar la configuración de esta matriz utópica. Entre ellas he encontrado las siguientes:

\subsection{Igualdad vital}

Las ordenaciones jurídicas modernas establecen la igualdad jurídica de todos los ciudadanos. Por ejemplo el Artículo 14 de la Constitución Española de 1978 establece la igualdad ante la ley, aunque luego puede ser pervertida mediante la pericia -o falta de ella- de un abogado. Moro, Bacon y Campanella vivieron bajo el dominio de la Iglesia Católica, que hace iguales a las personas en origen y destino (Creación y Juicio de Dios). Moro y Campanella observaron que esta igualdad no tenía traslado en la convivencia entre las personas y pensaron la igualdad de otra forma, no en el nacimiento, ni en la muerte, sino una igualdad vital, en la práctica diaria, en lo cotidiano. Moro, por ejemplo, lo soluciona haciendo el trabajo obligatorio (Moro, 1980, 62-63).

En la república imaginada por Campanella las fatigas se reparten por igual, ya que "todos los habitantes de la Ciudad del Sol se ejercitan en la técnica militar, la agricultura y el pastoreo. Todo ciudadano está obligado a conocer estas funciones, consideradas las más nobles..." (Campanella, 1980, 154). No se trata de un igualamiento, ya que las personas que más se esfuerzan por adquirir conocimiento, que es el criterio de valor en la ciudad-Estado, ascienden en su jerarquía social. Esa igualdad en las condiciones vitales deja suficiente libertad individual para que persigan sus ambiciones. No basta con decir que todas las personas somos iguales ante Dios o ante la Ley, sino que la República debe establecer medidas para que se haga efectivo en la práctica cotidiana.

Respecto a la igualdad de la mujer, Moro y Campanella estaban sujetos al escrutinio del Santo Oficio y limitados por el sentido común de su tiempo, recuérdese que no es hasta el primer tercio del siglo XX que se reconoce definitivamente el sufragio femenino. Campanella y Moro ya las considera iguales en los aspectos prácticos, como el trabajo y la guerra. Campanella parece que incluso tiene que excusarse ante el lector por ello: "Tampoco queremos hacer con ellas una República de amazonas. Solamente las reforzamos para que sirvan a la defensa y a la prole." (Campanella, 1980, 221).

Las propuestas prácticas de estas utopías pueden resultar desfasadas, pero la idea de que no es suficiente con una promulgación de la igualdad, sino que la configuración de la sociedad tiene que reflejar esa igualdad en la cotidianidad vital resulta sorprendentemente actual. Si la comunidad humana consigue conferirse una Constitución Universal todos los seres humanos de la tierra tendrán la misma consideración jurídica, que les dará en teoría los mismos derechos, pero no será más que papel mojado si esa igualdad no se refleja en la vida de las personas.

\subsection{Prevalencia de la racionalidad}

Moro describe un Estado construido según criterios racionales. Hay 54 ciudades en la isla, todas a la misma distancia, parecidas entre sí, con edificios equidistantes, con cuatro hospitales cada una. Además hay casas para campesinos en todo el país, a las que son enviados a vivir por turnos. También establece el número de personas que tiene cada familia. Todo en la isla está distribuido de forma racional para que sea lo más eficaz posible. Incluso hasta el trabajo es el suficiente para proveer las necesidades: "En esta isla, dividido el día en veinticuatro horas, se consagran seis al trabajo, tres por la mañana y otras tantas después de almuerzo" (Moro, 1980, 59). La racionalidad es el principio regulador. Todo se hace conforme a la razón y no por los impulsos del deseo, la ambición, o el orgullo. Usan, por ejemplo, las mismas vestiduras sencillas sin elementos diferenciadores, porque son las que resultan más eficaces.

Lo que parece que ya empezaba a vislumbrarse en el Renacimiento es que la razón puesta al servicio de las emociones genera injusticias y arbitrariedades. Moro condena la explotación de los campos para producir lana en Inglaterra, que 
despoja mediante tretas a los dueños y arrendatarios, que se ven obligados a vagar en la miseria, y consecuentemente al crimen y a un posterior ajusticiamiento de dudosa legitimidad. Además "con frecuencia los ricos procuran reducir el salario de los pobres, no sólo con fraudulentas prácticas, sino con leyes encaminadas a tal fin, de modo que por si poco fuera compensar con tal mezquindad a quienes el público tanto debe, se colorea de justicia semejante arbitrariedad mediante disposiciones que la legalizan." (Moro, 1980, 116).

Cuando se pone el orden al servicio de las emociones, se encuentra un modo de convertir en racional lo que en realidad proviene del deseo de los seres humanos. Se trata de un orden falible porque no emerge de la razón, sino de la emoción. Quizá por eso tanto Moro como Campanella imaginaron repúblicas en las que las emociones estaban al servicio de la razón. No se incentiva la ambición, ni la codicia, sino que estas emociones se transforman en servicio a la República, como en la ambición del conocimiento en La Ciudad del Sol, o el desprecio del oro con el que se producen las cadenas de los esclavos en Utopía.

\subsection{Esencialidad jurídica}

Tanto Moro como Campanella describen organizaciones políticas en las que se toman decisiones sobre cuestiones prácticas, pero no complican la estructura jurídica por la que se rigen. Por ejemplo, los utopianos "tienen las pocas leyes que su organización exige y condenan a las naciones que se rigen por múltiples leyes que, junto con sus comentarios, ocupan muchos gruesos volúmenes. No juzgan razonable obligar a los hombres a ceñirse a tan abultado cuerpo legal, $\tan$ oscuro que no puede ser leído y entendido por cada uno de sus súbditos (...) el juez pondera los argumentos y al sentenciar protege a los simples de ingenio contra los duchos en maquinaciones" (Moro, 1980, 91- 93).

Antes que compartir un sistema es preferible compartir su sentido. Los principios rectores de una sociedad no pueden ser una maraña de normas que sólo los técnicos conozcan, sino varios preceptos claros y transparentes para todas quienes comparten ese sistema jurídico. Campanella también nos dice que "las leyes de la Ciudad del Sol son pocas, breves, claras y están escritas en una tabla de bronce" (Campanella, 1980, 185).

Las normas jurídicas básicas de la matriz política global deberían ocupar lo justo para ser asequibles para cualquier miembro de la comunidad humana (una tabla de bronce) y ser aplicables en sí mismas y no mediante desarrollo posterior. Este principio de esencialidad consiste en alcanzar un acuerdo de mínimos en lo jurídico, evitando una maraña de leyes que se contradicen entre sí y que no acaban de ser efectivas.

\subsection{Organización modular}

Tanto en Utopía como en La Ciudad del Sol la organización política parte de unidades que a su vez conforman un módulo, la unión de varios módulos crean un módulo compuesto. En Utopía "cada treinta familias eligen, en forma anual, un magistrado (...) y por cada diez Sifograntes, con sus correspondientes familias, hay otro magistrado (...) Todos los Sifograntes, que suman doscientos, eligen al Príncipe en una lista de cuatro candidatos, designados por los habitantes de los cuatro sectores de la ciudad" (Moro, 1980, 58). En La Ciudad del Sol, en cambio, la modularidad se establece desde arriba hacia abajo. Al jefe supremo le asisten tres jefes Poder, Sabiduría y Amor, y cada triunviro tiene bajo sus órdenes a tres magistrados.

La modularidad es una consecuencia de los dos principios anteriores, prevalencia de la racionalidad y esencialidad jurídica. Un módulo se somete a los mismos principios en la parte como en la totalidad y responde a leyes racionales. Una construcción realizada por módulos resultará eficaz por la intercambiabilidad de sus partes y por la sencillez de su producción. Conjuntamente, al contrario de lo que pudiera parecer, una estructura modular permite la diversidad, tanto en la cantidad de configuraciones que permite como por la forma de ordenar las partes. Una estructura modular para un mosaico puede ser un opus reticulatum, en la que todas las teselas son iguales, pero también un opus signinum, en las que las piezas diferentes son integradas y armonizadas en el módulo.

La organización modular permite conciliar lo igual y lo diferente en un mismo sistema. De ahí que la organización política mundial podría ser una matriz, es decir, una estructura modular en la que se insertan otros módulos u organizaciones políticas. 


\subsection{Principio teórico comunitarista}

Tanto en Utopía como en La Ciudad del Sol se alude a una intuición del derecho natural en la que todo es común, ya que compartimos el origen y el destino como especie y las personas sólo pueden subsistir y progresar en comunidad. Moro argumenta que la propiedad privada debe ser eliminada porque es una barrera para la distribución de los recursos de forma justa, de forma que "hasta que se haya suprimido la propiedad no habrá distribución equitativa ni justa de las cosas ni será el mundo felizmente gobernado, pues mientras aquélla se mantenga la más amplia y, con mucho, la mejor parte de la humanidad estará sometida a una carga de pesares y ansiedades" (Moro, 1980, 47-48). Por su parte, Campanella estima que la pobreza y la riqueza en sus extremos envilecen al ser humano, mientras que la comunidad hace que sean ricos y pobres al mismo tiempo, de forma que se no tienen por qué envilecer (Campanella, 1980, 166-167).

Aunque ambos plantean la comunidad como una solución práctica, podría ser considerada -mediante en un salto de cinco siglos- una solución teórica, es decir, todas las cosas son comunes en origen pero la forma en la que los recursos son gestionados en la práctica es indiferente, siempre que responda a este principio. Público y privado serían entonces dos maneras de entender la responsabilidad sobre el bien común.

Esta idea permite todo el espectro de formas de propiedad sea posible sin conflicto entre sí, desde una comunidad radical como la que plantea Campanella hasta la total privatización. En esencia responden a una elección sobre la apariencia y el mecanismo de la propiedad temporal, pero asumiendo el principio teórico de que pertenecen a lo común. Si el origen común de toda propiedad es un incondicionado de su naturaleza, una forma de propiedad privada que atenta contra el interés de la Comunidad sería invalidada por derecho. En cambio, si lo incondicionado es la propiedad privada, la Comunidad está a expensas de ella, de su chantaje tácito y de que admita buenamente su participación en lo Común, ya que lo común se produciría por la suma de propiedades individuales.

\subsection{Sentido transcendental aglutinador}

En Utopía y La Ciudad de Sol existe una forma de trascendencia abierta, vaciada, en la que caben todos los conocimientos e ideas de otros lugares del mundo. En Utopía "hay diversas religiones no sólo en la isla, sino en cada ciudad. Unos rinden culto al Sol, a la Luna, o alguna estrella errante; otros, en cambio, adoran no como a una deidad ordinaria, sino como a la Suprema, a hombres que descollaron en otro tiempo por su virtud o su gloria. Pero la mayor parte de los utopianos rinde culto, en cambio, a una deidad eterna, invisible, infinita e incomprensible, que está más allá de lo que podemos conocer y se difunde por todo el universo (...) consideran impropio determinar a la ligera qué es verdad en religión, pues acaso los distintos credos sean todos inspirados por Dios que llega a los hombres en diferentes formas y se complace en tal diversidad, siendo por tanto indecente y demencial que unos amenacen y aterroricen a otros para hacerles creer en algo que no les parece cierto" (Moro, 1980, 103 -105). Campanella también describe una religión ecléctica en la que el hinduismo puede mezclarse con la doctrina pitagórica. Sin embargo, la religiosidad es lo que gobierna La Ciudad del Sol, como nos muestra el hecho de que el jefe supremo sea un sacerdote.

Lo que nos están diciendo ambos autores es que la religión debe servir para unir y no para separar, algo que en su tiempo ocurría muy al contrario, siendo motivo de guerras y enfrentamientos religiosos, uno de los cuales le costó la vida a Moro. Debería ser útil como fundamento de cohesión, tal como hicieron los padres de Estados Unidos, que se sirvieron de la religión como un aglutinador civil. Se llamaron una nación ante Dios, pero sin determinar qué Dios concreto. Dieron forma a una religión civil que estaba vaciada de dogmas, ya que convivía una diversidad de comunidades cuyas creencias no eran siempre coincidentes, pero con un mínimo en común, que sirvió de sentido transcendental aglutinador. La religión civil le confiere al individuo un vínculo moral con sus semejantes y un sentido en el desarrollo de su papel en la sociedad. (Sánchez-Bayón, 2008, 13).

La mera creencia en que la humanidad tiene un sentido más allá de sí misma, puede ser compartida tanto por el ateo, por el agnóstico, por el científico o por el religioso. Cada uno elige íntimamente en dónde sitúa ese sentido: en el conocimiento científico, en la ecología, en el desarrollo tecnológico, en la perfectibilidad humana o en Dios. El sentido transcendental aglutinador está abierto a todas las interpretaciones y desacuerdos, tanto religiosos como científicos, pero sitúa al individuo ante sí mismo y ante la Comunidad. 


\section{Conclusiones}

Los problemas a los que se enfrenta la comunidad humana requieren de una respuesta conjunta, pero la diversidad de poderes políticos está sometida a un único mecanismo socioeconómico que constriñe su efectividad. Por eso he planteado la tesis de una alternativa política en la que una matriz política pudiera aglutinar diferentes formas de organización socioeconómica. Ante la naturaleza utópica del propósito, he acudido a las utopías renacentistas para buscar propuestas.

Esta investigación se circunscribe a un ámbito teórico meramente especulativo, y dentro de esos límites he planteado algunas ideas del utopismo clásico que permitan armonizar la diversidad, la pluralidad y el desacuerdo en un único sistema matricial. Acuden a una igualdad vital, ya que la igualdad como condición puede ser pervertida fácilmente, y a la razón como criterio regulador del Estado. Además las normas jurídicas deberían ser aprehensibles por los ciudadanos. Estas dos ideas nos llevan a una organización modular, en la que la distribución es racional y las mismas leyes se aplican en las partes como en el todo.

Aunque Moro y Campanella proponen la eliminación de la propiedad privada, podemos interpretar la comunidad de bienes como un incondicionado que invalida cualquier forma de propiedad que no es capaz de satisfacer su naturaleza fundacional. Finalmente, es necesario un elemento aglutinador que dote de sentido a esta estructura matricial, que siguiendo a las utopías del Renacimiento, puede ser una idea de trascendentalidad vaciada de dogmas.

Este artículo ofrece al debate una lectura del utopismo clásico para enfrentar eficazmente los retos planetarios de nuestro tiempo. Conjuntamente, la hermeneusis ha permitido entender los textos renacentistas a la luz los problemas de nuestro tiempo y viceversa. Estas tres direcciones quedan abiertas para futuras investigaciones.

\section{Referencias}

HerRera Guillén, R. (2013). Breve historia de la utopía. Madrid: Nowtilus.

GILl, S. Y LAw, D. (1989), "Global Hegemony and the Structural Power of Capital" En: International Studies Quarterly, Volumen 33, Número 4.

Moro, T., Campanella Y T., Bacon, F. (1980). Utopías del Renacimiento (Utopía / La ciudad del Sol / Nueva Atlántida), Madrid: Fondo de Cultura Económica FCE.

SÁnCHEZ-BAYÓN, A. (2008-13). La Modernidad sin prejuicios. La religión en la vida pública estadounidense; (3 Volúmenes), Madrid: Ediberun/Delta Publicaciones. 